\title{
Natural history of peripheral neovascularisation in diabetic retinopathy
}

\author{
G S TURNER, D V INGLESBY, B SHARRIFF, AND E M KOHNER \\ From the Department of Medicine, Hammersmith Hospital, Du Cane Road, London W12 OHS
}

SUMMARY A study of 62 untreated eyes of 54 diabetic patients with peripheral neovascularisation is reported. The study shows that neovascularisation rarely remains confined to one sector of the retina; $18 \%$ of eyes had developed disc new vessels within one year, $27 \%$ had a vitreous haemorrhage within five years, and $10 \%$ developed either macular traction or a retinal detachment.

Peripheral neovascularisation in diabetic retinopathy is considered to have a benign prognosis as opposed to disc neovascularisation, in which the prognosis is unfavourable.' The present study was therefore conducted to determine the likely outcome, without treatment, in eyes with peripheral new vessels, which in contrast to disc new vessels (NVD) are also known as new vessels elsewhere (NVE). ${ }^{2}$ Events recorded were the progression of NVE in each retinal quadrant ('by sector'), the development of disc new vessels, vitreous haemorrhage, and retinal detachment.

\section{Patients and methods}

The study included 62 eyes of 54 diabetic patients with NVE, all of whom were attending the Hammersmith Hospital Diabetic Retinopathy Clinic during 1965-83. The criterion for inclusion was the clinical diagnosis of one or more areas of NVE, confirmed on colour photography and/or fluorescein angiography. Excluded were eyes with new vessels on or within one disc diameter of the optic disc, and those considered to have intraretinal microvascular abnormalities (IRMA) alone. Also excluded were eyes that had received photocoagulation or patients who had undergone pituitary ablation. Assessment was made by annual clinical examination and colour fundus photography, with fluorescein angiography at diagnosis and thereafter approximately biannually. Clinical details included best corrected visual acuity, slit-lamp biomicroscopy, and fundal examination by direct and indirect ophthalmoscopy, under mydriasis, by the staff of the Diabetic Retinopathy Clinic. Visual acuity was scored $1-12$ between $6 / 6$ on

Correspondence to Dr G S Turner, Moorfields Eye Hospital, City Road, London EC1V 2PD. the Snellen chart to no perception of light (NPL). Snellen acuities of $6 / 6-6 / 60$ were scored $1-7$, V/As $3 / 60-$ NPL were scored $8-12(8=3 / 60,9=$ counting fingers, $10=$ hand movements, $11=$ perception of light, $12=$ no perception of light). Photographic documentation was obtained by a method of standard photographic survey, as defined by Oakley et al. ${ }^{3}$ using a $30^{\circ}$ field of the Zeiss (Oberkocken) fundus camera. For the purpose of this assessment the retina was divided into four sectors (upper temporal, lower temporal, upper nasal, lower nasal) by a horizontal and vertical line bisecting the optic disc. Where a single area of neovascularisation was crossed by these lines, grading was limited to the predominant sector affected. Progression of NVE was assessed by the development of new vessels in separate sectors of the retina. Grading change in a single area or the development of a separate area within the same sector was not considered. Most patients were studied prior to the value of photocoagulation in diabetic retinopathy being established.

There were 29 males $(54 \%)$ and 25 females $(46 \%)$. Thirty-one $(57 \%)$ had insulin dependent diabetes (IDDM) and $23(43 \%)$ had non-insulin dependent diabetes (NIDDM). The mean duration of diabetes to diagnosis of NVE in those with IDDM was $16 \cdot 6$ years (SD 8.8; range 4-41), with mean age of onset of diabetes 21.8 years (SD 10.5; range 5-46). In those with NIDDM, the duration of known diabetes to NVE formation was $7 \cdot 2$ years (SD 6.5; range $0-25$ ), with a mean age of onset $50 \cdot 1$ years (SD $8 \cdot 3$; range 32-62). The mean duration of follow up was 4.8 years (SD 2.6; range 1-11). Twenty-nine eyes of 28 patients were followed up for a period of at least five years. Among the remaining 26 patients the period of observation was terminated by death in 12 patients 
Table 1 Percentage of eyes, grouped according to number of sectors containing NVE, at each year of follow-up

\begin{tabular}{lcccccc}
\hline $\begin{array}{l}\text { No. of } \\
\text { sectors }\end{array}$ & \multicolumn{1}{l}{ Year } & & & & \\
\cline { 2 - 6 } & 0 & 1 & 2 & 3 & 4 & 5 \\
\hline 1 & 45 & 24 & 20 & 18 & 17 & 17 \\
2 & 26 & 31 & 27 & 23 & 27 & 15 \\
3 & 24 & 32 & 31 & 16 & 17 & $29 \cdot 5$ \\
4 & 5 & 11 & 6 & 14 & 100 & $29 \cdot 5$ \\
ng & 0 & 100 & 100 & 100 & & 100 \\
Total & 100 & & & & & 100 \\
\hline
\end{tabular}

$\mathrm{ng}=$ Not gradable owing to complications, for example, vitreous haemorrhage.

(14 eyes) after a mean follow up $2 \cdot 8$ years (SD $1 \cdot 2$; range 1-4), and four patients (four eyes) were lost to follow-up after a mean of 1.5 years (SD 0.6 ; range $1-3)$ years. As a result of reports concerning the efficacy of photocoagulation ${ }^{24}$ it was considered unethical to withhold this treatment after 1978 in eyes with progressive retinopathy. Thus 15 eyes of 14 patients (of whom four died before five years of follow-up was completed) were treated after a mean follow-up of 2.7 years (SD 1.2 ; range 1-4), and thereafter excluded from the present study.

\section{Results}

PROGRESSION OF NVE

A steady progression of NVE in other areas of the retina is demonstrated in Table 1 . Of the 28 eyes with one sector alone involved at diagnosis in only two
$(7 \%)$ did the new vessels remain limited to that sector after five years, although in an additional two (7\%) eyes the new vessels had regressed. NVE had developed in at least one further sector in 15 of $28(54 \%)$ within one year, and at five years seven of $16(44 \%)$ had new vessels in four sectors or were ungradable owing to complications. Of the whole group 44 of 62 $(71 \%)$ at diagnosis had NVE confined to one or two sectors only, progressing to 16 of $27(59 \%)$ with NVE in four sectors or ungradable owing to complications at five years (Fig. 1).

\section{DEVELOPMENT OF DISC NEW VESSELS}

In the whole group 24 out of 62 eyes ( $39 \%$ ) developed disc new vessels (NVD) after a mean duration of follow-up of 2.3 years (SD 1.3; range 1-6) from diagnosis of NVE alone (Fig. 2; Table 2). At one year NVD had already developed in 11 eyes $(18 \%)$. NVE

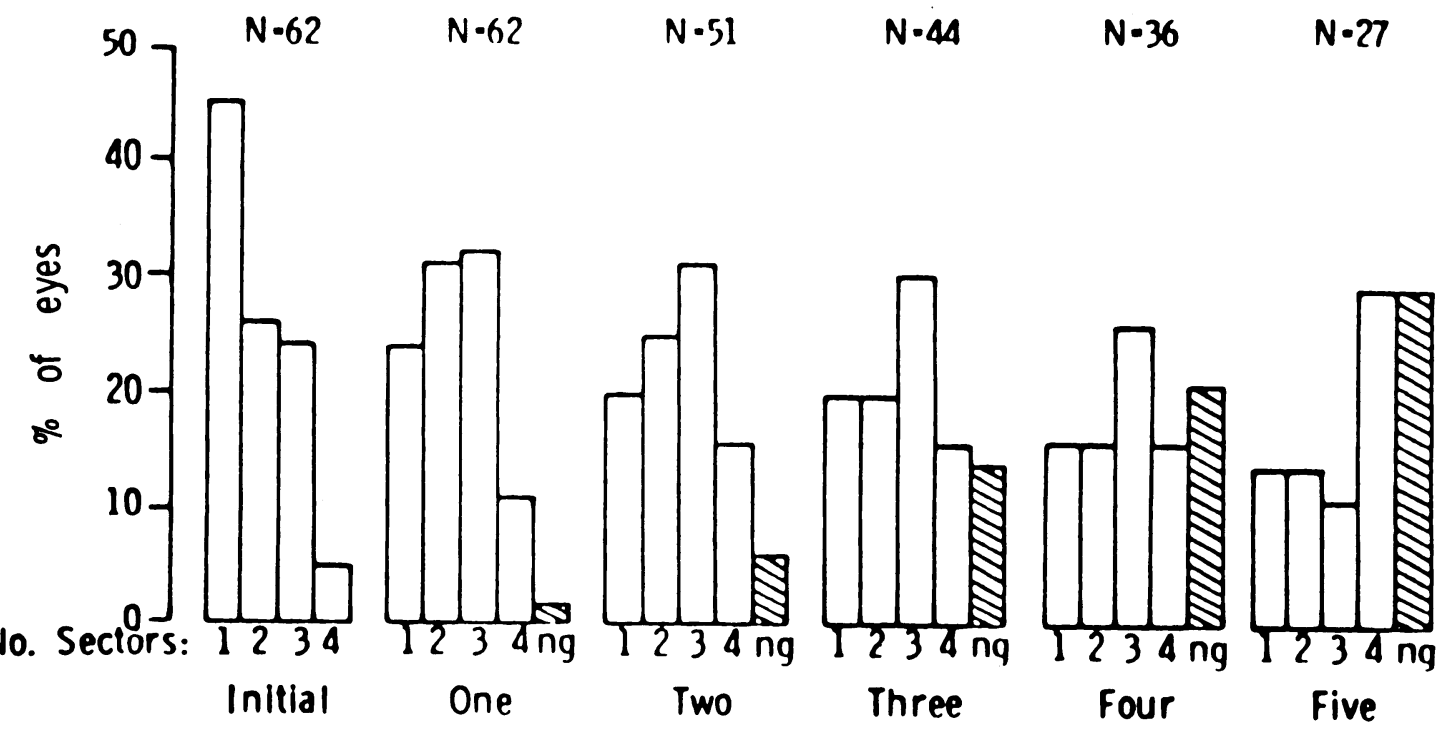

Years from diaqnosis of NVE

Fig. 1 The number of sectors containing new vessels elsewhere (NVE) at each year of follow-up expressed as a percentage of the whole group (ng=not gradable). 
Fig. 2 The percentage of eyes developing disc new vessels (NVD) at each year of follow-up (five $+=$ five or more).

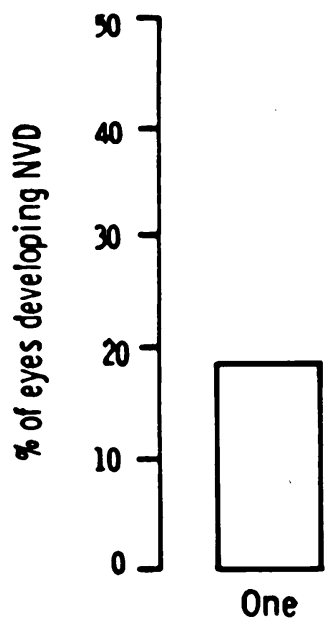

Fig. 3 The percentage of eyes developing a vitreous haemorrhage at each year of follow-up (five $+=$ five or more).
VITREOUS HAEMORRHAGE

Vitreous haemorrhage (VH) occurred in 17 eyes $(27 \%)$ after a mean follow-up of 2.8 years (SD 1.5; range 1-7) (Fig. 3, Table 3). The mean age at the time

Table 3 Occurrence of vitreous haemorrhage

\begin{tabular}{cccccc}
\hline \multicolumn{5}{c}{ Year of follow-up } \\
\cline { 2 - 6 } & 1 & 2 & 3 & 4 & $5+$ \\
\hline $\begin{array}{c}\text { No. of eyes } \\
\text { with VH } \\
\% \begin{array}{c}\text { of eyes } \\
\text { with VH }\end{array}\end{array}$ & 5 & 9 & 12 & 13 & 17 \\
\hline
\end{tabular}

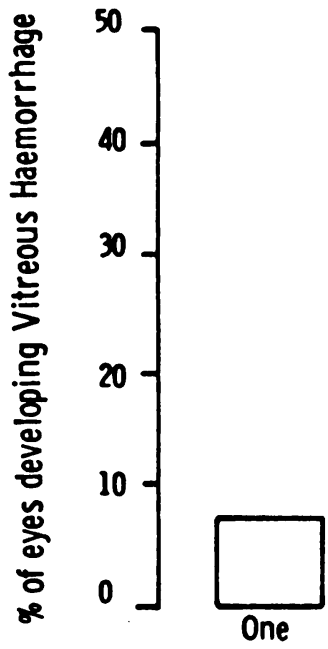

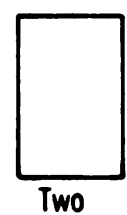
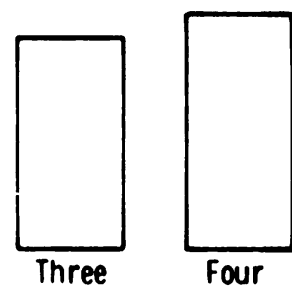

Four

Five +

Years from diagnosis of NVE 
of haemorrhage was 51.8 years (SD 13.8; range 29-75). The visual acuity was reduced by a mean score of 3.7 in 15 of $17(88 \%)$. Of these, $60 \%$ ( 9 of 15 ) made no spontaneous visual recovery, and $40 \%$ (6 of $15)$ recovered a mean score of $2 \cdot 7$, within one year. Within a further year three of these had lost vision owing to rebleeding. In two eyes $(12 \%) \mathrm{VH}$ occurred without an objective deterioration in the visual acuity. Those with localised subhyaloid haemorrhage were not included. At the time of haemorrhage seven of $17(41 \%)$ had NVE alone, whereas 10 of $17(59 \%)$ had developed NVD.

\section{FIBROSIS; MACULAR TRACTION; RETINAL DETACHMENT}

The development of fibrous tissue in association with NVE occurred in 25 of $62(40 \%)$ eyes, after a mean follow-up of 2.3 years (SD 2.2; range $0-3$ ) from diagnosis of NVE. Of the whole group eight of 62 $(13 \%)$ developed tractional complications; of these, three of eight $(37 \%)$ had macular traction alone, and five of eight $(63 \%)$ developed a retinal detachment. No distinction could be made between rhegmatogenous and traction detachment alone, and details of the extent and rate of progression have not been investigated. The mean follow-up from diagnosis of NVE was 3.7 years (SD 1.4; range 2-6), with a mean duration of associated fibrous tissue, before detachment, of 2.5 years (SD 1.4; range $1-5$ ).

\section{VISUAL ACUITY}

Forty-two of 62 eyes $(68 \%)$ started the study with a visual acuity of $6 / 6$ or better. In 13 of $62(21 \%)$ vision was between $6 / 9$ and $6 / 18$, and in seven eyes $(11 \%)$ it was between $6 / 24$ and $6 / 60$. Initial visual loss was attributable to diabetic macular disease in 18 of 20 $(90 \%)$, owing to lens opacity in one $(5 \%)$ and amblyopia in one $(5 \%)$. After a mean follow-up of 4.8 years (SD 2.6; range 1-11) acuity had deteriorated in 27 eyes $(44 \%)$ by a mean score of 4.3 (SD $2 \cdot 6$ ). Acuity was then $6 / 6$ or better in 29 of 62 eyes $(47 \%)$, between $6 / 9$ and $6 / 18$ in 11 eyes $(18 \%)$, between $6 / 24$ and $6 / 60$ in nine eyes $(14 \%)$, and worse than $6 / 60$ in 13 eyes ( $21 \%$ ) (Fig. 4). Of the 27 eyes which deteriorated, visual loss was attributed to diabetic macular disease in $13(49 \%)$, to vitreous haemorrhage alone in five (18\%), to tractional complications with or without vitreous haemorrhage in eight $(29 \%)$, and to thrombotic glaucoma with vitreous haemorrhage in one $(4 \%)$.

\section{Discussion}

The predominance of proliferative retinopathy in younger diabetics with IDDM is reflected in the study population $(57 \%)$. It is, however, notable that $43 \%$ had NIDDM with a mean age of onset of $50 \cdot 1$ years, confirming the increasingly recognised fact that neovascular retinopathy is also important in this older age group. ${ }^{67}$ That new vessels are diagnosed after a shorter duration of known diabetes in patients with NIDDM (a mean of 7.2 years after diagnosis of diabetes as opposed to 21.7 years in those with IDDM) may reflect a prolonged subclinical disease state in the former group.

In the group of patients studied neovascularisation

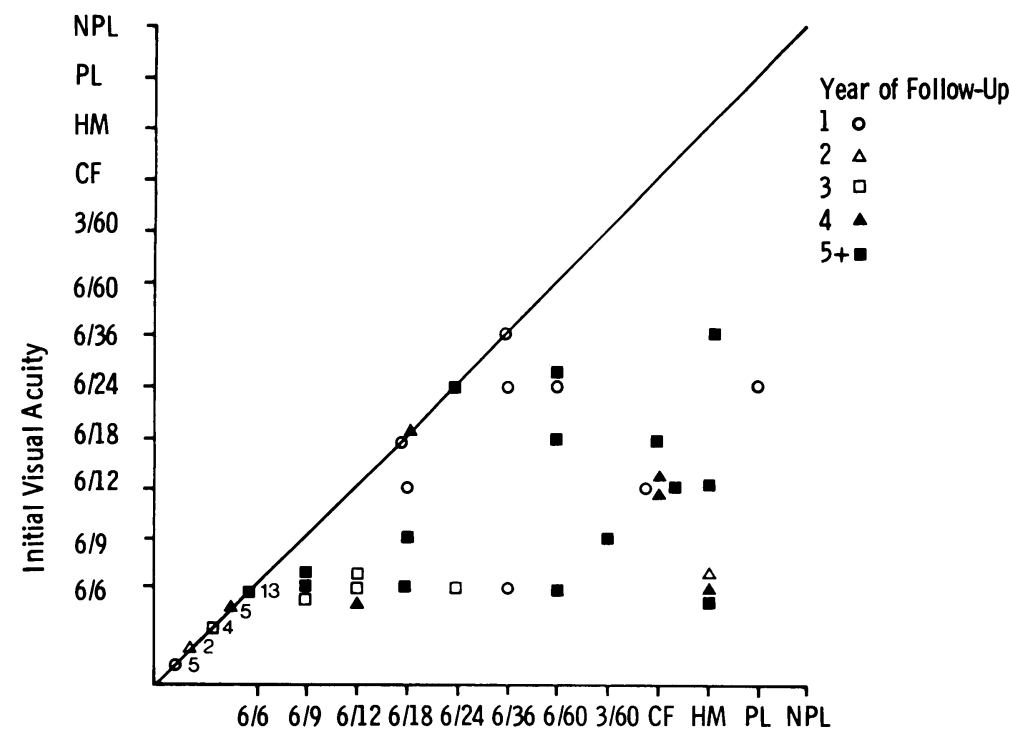

Fig. 4 Initial and final visual acuity. Eyes are represented by a symbol denoting duration of follow-up in years $(5+=5$ or more $)$, individually, or grouped with the number in each indicated. Diagonal: No change line. $C F=$ counting fingers. $H M=$ hand movements. $P L=$ perception of light. $N P L=$ no perception of light .

Final Visual Acuity 
rarely remained confined to the initial sector or sectors affected. This presumably reflects the generalised nature of the underlying microangiopathy and resultant capillary closure. It was not possible to study the extent of peripheral capillary closure, as initial fluorescein angiograms did not incorporate a peripheral survey. This progression implies that local or sector treatment may not be adequate, particularly where careful follow-up cannot be assured.

The development of NVD in $18 \%$ of eyes at one year is similar to that found in a comparable group of eyes by the Diabetic Retinopathy Study Research Group. ${ }^{4}$ In the control group of eyes with NVE alone in the British Multicentre Trial of Photocoagulation ${ }^{8}$ $50 \%$ developed NVD within five years as opposed to $37 \%$ in the present study. These two controlled clinical trials ${ }^{\mathrm{x} \varphi}$ have demonstrated unequivocally the benefits of photocoagulation for NVD, though the results for treatment for NVE alone have been less clear. The British Multicentre Group ${ }^{8}$ were unable to demonstrate a statistically significant difference between control and treated eyes with NVE alone over a five-year period, despite the high percentage that developed NVD. In the USA the Diabetic Study Research Group ${ }^{9}$ could not recommend a clear choice between prompt photocoagulation and deferral in this group of patients, provided high-risk characteristics ${ }^{4}$ such as preretinal or vitreous haemorrhage were not present. In the present study $27 \%$ of eyes developed a vitreous haemorrhage, of which $50 \%$ did not make any spontaneous visual recovery, $40 \%$ developed fibrosis associated with NVE, and $10 \%$ developed retinal tractional complications, when left untreated. Why there should be no difference between treatment and no treatment in the British study, especially when $50 \%$ of the control group developed NVD, is not clear, but it is probably due to the fact that, after 1977, eyes that developed NVD in the control group were treated.

The present study indicates that peripheral neovascularisation can result in serious complications, which may preclude photocoagulation. Therefore treatment should probably be undertaken immediately, unless frequent follow up can be assured. Some authorities advocate focal or sectorial treatment initially ${ }^{10}$ associated with careful follow-up. In view of the likely development of NVE in other sectors, and the greater recurrence of NVE following sector treatment as opposed to panretinal photocoagulation (PRP), " the latter may be more appropriate, particularly if follow-up cannot be assured.

The authors acknowledge the work of the ophthalmologists and photographers who worked in the unit during the period of management of the patients in the study.

This work was supported by a Group Grant from the Scientific Section of the British Diabetic Association.

\section{References}

1 Deckert T, Simonsen SE, Poulsen JE. Prognosis of proliferative retinopathy in juvenile diabetes. Diabetes 1967; 16: 726-33.

2 The Diabetic Retinopathy Study Research Group. Preliminary report on the effects of photocoagulation therapy. Am J Ophthalmol 1976; 81: 383-96.

3 Oakley NW, Hill DW, Joplin GF, Kohner EM, Fraser TR. Diabetic retinopathy. 1 . The assessment of severity and progress by comparison with a set of standard photographs. Diabetologia 1967; 3: 402-5.

4 The Diabetic Retinopathy Study Research Group. Photocoagulation treatment of proliferative diabetic retinopathy: the second report of Diabetic Retinopathy Study Findings. Ophthalmology (Rochester) 1978; 85: 82-106.

5 Bodansky HJ, Cudworth AG, Whitelocke RAF, Dobree JH. Diabetic retinopathy and its relation to type of diabetes: review of a retinal clinic population. BrJ Ophthalmol 1982; 66: 496-9.

6 British Multicentre Study Group. Photocoagulation for diabetic maculopathy: a randomised controlled clinical trial using the xenon-arc. Diabetes 1984; 32: 1010-6.

7 Klein R, Klein B, Moss S, Davis M, DeMets D. The Wisconsin Epidemiologic Study of Diabetic Retinopathy: III: Prevalence and risk of diabetic retinopathy when age at diagnosis is $\mathbf{3 0}$ or more years. Arch Ophthalmol 1984; 102: 527-32.

8 British Multicentre Study Group. Photocoagulation for proliferative diabetic retinopathy: a randomised controlled clinical trial using the xenon-arc. Diabetologia 1984; 26: 109-15.

9 The Diabetic Study Research Group. Photocoagulation treatment of proliferative diabetic retinopathy. Diabetic Retinopathy Study Research Group report No. 8. Ophthalmology (Rochester) 1981; 88: 583-600.

10 Hamilton AMP, Blach RK, Whitelocke R. Management of proliferative diabetic retinopathy. In: Kanski JJ, Morse PJ, eds. Disorders of the vitreous retina and choroid. London: Butterworths, 1983: 104-6.

11 Inglesby DV, Turner GS, Schulenburg WS, Kohner EM. Photocoagulation for peripheral neovascularisation in diabetes. BrJ Ophthalmol 1985; 69: 157-61. 